\title{
Hippocampal Activated Microglia May Contribute to Blood-brain Barrier Impairment and Cognitive Dysfunction in Post-traumatic Stress Disorder-like Rats
}

\section{Kun Ni}

Nanjing Drum Tower Hospital: Nanjing University Medical School Affiliated Nanjing Drum Tower Hospital

Jixiang Zhu

Nanjing Drum Tower Hospital: Nanjing University Medical School Affiliated Nanjing Drum Tower Hospital

\section{Xuan Xu}

Nanjing Drum Tower Hospital: Nanjing University Medical School Affiliated Nanjing Drum Tower Hospital

\section{Yue Liu}

Nanjing Drum Tower Hospital: Nanjing University Medical School Affiliated Nanjing Drum Tower Hospital

\section{Shuai Yang}

Nanjing Drum Tower Hospital: Nanjing University Medical School Affiliated Nanjing Drum Tower Hospital

\section{Yulin Huang}

Nanjing Drum Tower Hospital: Nanjing University Medical School Affiliated Nanjing Drum Tower Hospital

\section{Rui Xu}

Nanjing Drum Tower Hospital: Nanjing University Medical School Affiliated Nanjing Drum Tower Hospital

\section{Li Jiang}

Nanjing Drum Tower Hospital: Nanjing University Medical School Affiliated Nanjing Drum Tower Hospital

\section{Juan Zhang}

Nanjing Drum Tower Hospital: Nanjing University Medical School Affiliated Nanjing Drum Tower Hospital

\section{Wei Zhang}

Nanjing Drum Tower Hospital: Nanjing University Medical School Affiliated Nanjing Drum Tower Hospital 


\section{Zhengliang Ma ( $\triangle$ mazhengliang1964@163.com )}

Nanjing University Medical School Affiliated Nanjing Drum Tower Hospital https://orcid.org/00000003-3173-2944

\section{Research Article}

Keywords: Post-traumatic stress disorder, Single prolonged stress, Blood-brain barrier, Cognition dysfunction

Posted Date: November 15th, 2021

DOI: https://doi.org/10.21203/rs.3.rs-1055193/v1

License: (c) (i) This work is licensed under a Creative Commons Attribution 4.0 International License. Read Full License

Version of Record: A version of this preprint was published at Journal of Molecular Neuroscience on February 15th, 2022. See the published version at https://doi.org/10.1007/s12031-022-01981-4. 


\section{Abstract}

Post-traumatic stress disorder (PTSD) associated cognitive dysfunction significantly disturbs patients' quality of life and will-to-live. However, its underlying mechanism is as yet unknown. Recent researches indicate blood-brain barrier (BBB) breakdown is responsible for early cognitive dysfunction. Microglia might participate in remodeling of BBB-associated tight junction and regulating BBB integrity. Nevertheless, it is unclear whether microglia activation and BBB injury involve in PTSD-associated cognitive dysfunction. Hence, we established an animal model of PTSD, single prolonged stress (SPS) and investigated permeability changes in the hippocampus, and further explored the effects of microglia on BBB remodeling. The $Y$ maze was used to assess the changes of cognitive function. The sodium fluorescein ( $\mathrm{NaFlu}$ ) assay and western blotting analysis were employed to detect BBB integrity changes. Minocycline was administrated to inhibit microglial activation. Immunofluorescence stains were used to assess the activation states in microglia. The results showed that SPS exposed rats exhibited poorer cognitive performance, higher passage of NaFlu, and lower expression of tight junction proteins (occludin and claudin 5) in the hippocampus on the day after SPS, but no difference on the 7th day. Inhibition of microglial activation by minocycline attenuated poor cognitive performance and BBB impairment including the extravasation of NaFlu and protein levels of the tight junction. Taken together, the present study indicates that BBB impairment may underlie the shared pathological basis of PTSD and cognitive dysfunction. Microglial activation may involve in BBB remodeling at the early stage of SPS.

\section{Introduction}

Post-traumatic stress disorder (PTSD) is a complex and debilitating illness triggered by terrifying events, which is often companies by cognitive impairment, chronic pain, and affective disorders (Compean and Hamner 2019). The prevalence of PTSD in the general population is estimated at approximately $7 \%$ and higher among people experienced major traumatic events before (Zhou et al. 2021). For example, coronavirus disease-2019 (COVID-19) is a major source of traumatic stress for people around the globe since 2019. A meta-analysis revealed that the prevalence of PTSD among COVID-19 patients in the postillness stage was up to $32.2 \%$ (Rogers et al. 2020). PTSD with negative alterations in cognition and mood was markedly associated with high morbidity and disability (Brown et al. 2020). Although cognitive dysfunction in PTSD patients has proved to be a non-negligible clinical problem, its underlying mechanism remains to be elucidated.

Blood-brain barrier (BBB) is the structural and functional interface that regulates substance and energy exchange in the central nervous system (CNS) thus maintaining the homeostasis of CNS (Villaseñor et al. 2019). It is mainly composed of brain microvascular endothelial cells, glial end-feet, pericytes, and the extracellular matrix that form the neurovascular unit with the adjacent neurons, astrocytes, and microglia (ladecola 2017). Tight junctions between brain microvascular endothelial cells form the main anatomical basis of BBB and play an important role in limiting paracellular permeability (Rajagopal et al. 2019). Clinical studies showed that BBB breakdown in the hippocampus was a crucial characteristic of individuals with early cognitive dysfunction (Bowman et al. 2018; Nation et al. 2019; Montagne et al. 
2020). Animal experiments also revealed that stress such as sleep deprivation and restraint stress could induce BBB hyper-permeability which was associated with emotional-cognitive disorder (Xue et al. 2019; Sántha et al. 2015; Xu et al. 2019). These researches prompt BBB injury might be a key mechanism of cognitive dysfunction.

Microglia is the rapid response cells coping with central nervous system injury including PTSD. Our previous study found that single prolonged stress (SPS), an animal model of PTSD, induced anxiety-like behavior and was accompanied by the activation of microglia in the CA1 and CA3 regions of rat hippocampus, which could be reversed by prior administration of microglia inhibitor (minocycline) (Sun et al. 2016). Recent researches suggested microglia might contact with neuro-vasculature and crosstalk with endothelial to participate in remodeling of BBB-associated tight junction and regulating BBB integrity (Bisht et al. 2021; Delaney et al. 2021). Nevertheless, it is unclear whether microglia activation and BBB injury involve in PTSD-induced cognitive dysfunction. Therefore, we established SPS-induced cognitive dysfunction and investigated permeability changes in the hippocampus, and further explored the effects of activated microglia on BBB remodeling.

\section{Materials And Methods}

\section{Experimental animals}

Adult male Sprague-Dawley rats (210-230 g) were obtained from Charles River Animal Company (Zhejiang, China). Rats were housed in the barrier environment with free access to the diets and drinking water, light on between 8:00 and 20:00 hours. All rats were allowed to acclimate to their environment for at least 1 week before the experimental procedure. All the experimental protocols were approved by the Ethics Committee of Drum Tower Hospital.

\section{Experimental schemes}

Experiment 1. 36 rats were randomized into two groups $(n=18)$ : group $C$ (rats with no treatment) and group SPS (rats suffered from SPS). Nine rats were randomized picked to perform Y maze test on day 1 and day 7 respectively after SPS treatment. After the behavior test, the brain samples were collected for western blot analysis and BBB permeability assessment.

Experiment 2. $36 \mathrm{SD}$ rats were randomly assigned into 4 groups, group $\mathrm{C}+\mathrm{V}$ (control+vehicle, rats treated with saline), group $\mathrm{C}+\mathrm{M}$ (control+minocycline, rats treated with minocycline), group S+V (SPS+vehicle, SPS treated rats injected with saline), and group S+M (SPS+minocycline, SPS treated rats injected with minocycline) ( $n=9 /$ group). Rats received minocycline (30 mg/ $\mathrm{kg}$ ) or an equal volume of saline intraperitoneally $30 \mathrm{~min}$ before and $23 \mathrm{~h}$ after SPS. Doses of systemic minocycline treatment were employed according to our previous study showing the potent inhibitory effect on activated microglia (Sun et al. 2016). The behavior test was conducted on the day after SPS. After that, the brain samples were collected for immunofluorescence $(n=3)$, western blot analysis $(n=3)$, and BBB permeability assessment $(n=3)$. 


\section{SPS procedure}

The SPS procedure referred to a previous report (Sun et al. 2016). Rats were immobilized inside a plastic container for $2 \mathrm{~h}$ and then forced to swim in the clean water $\left(24^{\circ} \mathrm{C}\right)$ for $20 \mathrm{~min}$. After swimming, the rats were dried and allowed a 15 min break. Finally, rats were anesthetized with ether until consciousness disappearance. Meanwhile, control rats were kept in the next room without any treatment.

\section{Y maze test}

Spontaneous alternation was used to assess the working memory in $\mathrm{Y}$ maze test (Li et al. 2019; Duchon et al. 2020). The $Y$ maze consists of three arms $(50 \mathrm{~cm} \times 10 \mathrm{~cm} \times 30 \mathrm{~cm})$ at $120^{\circ}$ angles to each other. After habituating the behavior test room for $1 \mathrm{~h}$, rats were transferred to the center of $\mathrm{Y}$ maze to explore for 8 min freely. A video-tracking system was employed to record activity trajectories and the series of arm entries. Spontaneous alternation percentage was estimated by EthoVision XT software (Noldus information technology, Wageningen, Netherlands). Three consecutive entries into three different arms were defined as a success. Spontaneous alternation (\%) $=$ [(number of alternations)/ (total arm entries-2) $]$ $\times 100 \%$.

\section{BBB permeability assessment}

BBB permeability was estimated by the sodium fluorescein (NaFlu) assay (Yang et al. 2014). Briefly, $10 \%$ $\mathrm{NaFlu}$ (Sigma Aldrich, $2 \mathrm{ml} / \mathrm{kg}$ ) was injected via the tail vein and allowed to circulate for $30 \mathrm{~min}$. Subsequently, cold saline was perfused transcardially for $15 \mathrm{~min}$ to remove the intravascular dye after anesthesia. The hippocampus was sampled and weighed. Samples were homogenized in $500 \mu \mathrm{l}$ PBS and vortexed thoroughly after adding equal volumes of $60 \%$ trichloroacetic. Samples, cooled for 30 min, were centrifuged at $14,000 \times \mathrm{g}$ for 10 min later. The concentration of NaFlu was detected (excitation wavelength, $440 \mathrm{~nm}$; emission wavelength, $525 \mathrm{~nm}$ ). A standard curve was drawn to link optical density and the concentration of NaFlu. BBB permeability was determined by the weight of NaFlu per gram of tissue.

\section{Immunofluorescence}

Rats were transcardially perfused with cold saline under deep anesthesia and then fixed with $10 \%$ buffered formalin phosphate. Whole brains were quickly removed and post-fixed overnight. Fixed samples were dehydrated in a gradient concentration of sucrose. Twenty- $\mu \mathrm{m}$ frozen sections were balanced at room temperature for $15 \mathrm{~min}$ and washed in PBS for $5 \mathrm{~min}$ thrice. Sections were blocked with $10 \%$ goat serum for $1 \mathrm{~h}$ at room temperature. subsequently, they were incubated with primary antibodies anti-lba1 (1:500, Wako) overnight at $4{ }^{\circ} \mathrm{C}$ and were washed three times in PBS. Secondary antibodies, conjugated to AlexaFluor 488, were applied to slides to incubate for $1 \mathrm{~h}$ at room temperature. Stains were washed three times with PBS before nuclear staining with DAPI (Life Technologies). Microglial activation was reflected by comparing the numbers of Iba1-positive cells between groups. The numbers of Iba1positive cell bodies, colocalized with DAPI, were counted manually (Sun et al. 2016). 


\section{Western blotting}

Western blotting was performed as described previously (Wang et al. 2020). Total protein from hippocampus was extracted by adding the ice-cold RIPA Lysis Buffer. Proteins were separated by gradient electrophoresis on 10-12.5\% SDS-PAGE gels and then transferred onto a polyvinylidene fluoride membrane (Millipore, USA). After they were blocked in $5 \%$ skim milk, the membranes were incubated with the following primary antibodies overnight at $4{ }^{\circ} \mathrm{C}$ : claudin 5 (1:1000, Thermo Fisher, USA), occludin (1:10,000, Proteintech, China), Iba1 (1:500, Wako, Japan), and GAPDH (1:10,000, Proteintech, China). After washes with TBST, the corresponding secondary antibodies were applied for $1 \mathrm{~h}$ at room temperature (1:10000, Millipore, USA). membranes were visualized using ECL solution.

\section{Statistical analysis}

Data are presented as the means \pm SD. Nonparametric Student's t-test was used to compare the differences between groups. One-way ANOVA was performed to determine differences in results among groups. The statistical analysis was conducted using SPSS 20.0 software (IBM, Armonk, USA). $P<0.05$ was considered as statistically significance.

\section{Results}

\section{SPS induced cognitive impairment}

Spontaneous alternation test was performed in $\mathrm{Y}$ maze to assess the cognitive function. The typical movement trails of rats on day 1 and 7 after SPS were shown in Fig. 1a. Student's t-test revealed a significant difference in Y-maze spontaneous alternation of group SPS and group C on the day after SPS $[(50.38 \pm 9.97) \%$ vs. $(64.06 \pm 8.82) \%, t=2.55, P<0.01]$ (Fig. 1 b). However, there was no statistically significant difference between two groups at the 7 th day $[(65.17 \pm 11.13) \% v s$. $(66.97 \pm 6.46) \%, t=0.42 \rrbracket$ $P>0.05]$ (Fig. 1b).

\section{SPS induced hippocampal BBB impairment}

To determine the changes of hippocampal BBB permeability, we detected the passage of NaFlu and levels of endothelial tight junction proteins of rats in group SPS and group C. NaFlu assay showed that the extravasation of NaFlu is higher in group SPS than in group $C$ on 1 st day after SPS $[(0.93 \pm 0.06) \mathrm{ug} / \mathrm{g}$ vs. $(0.61 \pm 0.10) \mathrm{ug} / \mathrm{g}, t=4.53, P<0.05]$, while no statistical difference was found on 7 th day (Fig. 2a). Western blotting analysis revealed that the expression levels of tight junction protein occludin $[(0.76 \pm$ $0.20)$ vs. $(1.17 \pm 0.15), t=2.87, P<0.05]$ and claudin 5 [(0.30 \pm 0.08$)$ vs. $(1.23 \pm 0.16), t=9.08, P<0.001]$ significantly decreased in group SPS (Fig. $2 b$ ).

\section{Inhibition of microglial activation attenuated cognitive impairment}

To investigate the effect of hippocampal activation of microglia in the pathogenesis of PTSD associated cognitive dysfunction, minocycline was employed as its potential inhibition of microglial activation in the 
following experiment. Minocycline hydrochloride (30 mg/kg, i.p.) or the same volume of vehicle was injected at $30 \mathrm{~min}$ before and on the day after SPS to control and SPS exposed rats. Spontaneous alternation was measured on the day after SPS. The typical trajectory of each group rats on day 1 after SPS were shown in Fig. 3a. Compared to group $S+V$, minocycline improved the spontaneous alternation performance of rats exposed to SPS $(P<0.01$, Fig.3b). However, minocycline had no effect on cognitive behaviors in control rats $(P>0.05$, Fig. 3b). Immunofluorescence was used to validate the morphological changes of microglia after SPS exposure. The number of positive stained cells of Iba1 was calculated and summarized in Fig. 3c. Rats exposed to SPS presented an increased number of activated microglia in the hippocampus $(P<0.001$, Fig. 3c), which were inhibited by minocycline $(P<0.001$, Fig. 3c). The representative micrographs for Iba1 in the $\mathrm{CA} 1$ and $\mathrm{CA} 3$ regions of rat hippocampus in each group were shown in Fig. 3d.

\section{Inhibition of microglial activation attenuated SPS induced BBB injury}

To assess whether microglial activation involves in BBB breakdown, NaFlu assay and western blot analysis were conducted. Hippocampal content of fluorescein sodium reduced after pretreated with minocycline in SPS exposed groups $[(0.54 \pm 0.04) \mathrm{ug} / \mathrm{g}$ vs. $(1.06 \pm 0.06) \mathrm{ug} / \mathrm{g}, q=10.06 \otimes P<0.001]$ (Fig. 4a). Compared with group $S+V$, decreased expression levels of occludin and claudin 5 were relieved by minocycline administration [(1.06 \pm 0.13$)$ vs. $(0.83 \pm 0.03), q=3.59 \otimes P<0.05 ;(0.71 \pm 0.10)$ vs. $(0.42 \pm 0.17)$, $q=2.95 \varangle P<0.05$ ] (Fig. 4b). Minocycline had no effect on BBB breakdown in control group both in NaFlu assay and western blot analysis $(P>0.05$, Fig. 4$)$.

\section{Discussion}

Our findings show that rats exhibit working memory impairment at the early stage after SPS. In the meantime, BBB integrity injury and microglial activation in the hippocampus were observed. Pretreated with minocycline, an inhibitor of activated microglia, not only inhibited the activation of microglia but also improved the hippocampal BBB disruption. Likewise, poor working memory in SPS treated rats was also attenuated by minocycline. Our results suggest that the activated microglia may contribute to SPSinduced BBB remodeling and working memory impairment.

Compared to the control group, the percentage of spontaneous alternation was significantly decreased on the first day after SPS. But no obvious difference was observed between groups on day 7 . The behavior results indicate that SPS may induce working memory impairment at the early stage, which is consistent with other research (Han et al. 2015). Meanwhile, the content of NaFlu and the protein levels of occludin and claudin 5 in the hippocampus showed markedly increasing and decreasing respectively in SPS group. These suggest that the integrity of hippocampal BBB was impaired in SPS condition. As another animal model of strong psychological stress, restraint stress treated rats also performed decreased expression and discontinuity of the occludin and claudin 5 in the hippocampus (Sántha et al. 2015). A network-level analysis of the hippocampus in the social defeat model for evaluating PTSD symptoms showed notable damage in tight junctions as well (Yang et al. 2013). Clinical observations provided 
analogous clues that the increased level of S-100 $\beta$ (a biomarker of BBB disruption) in the serum was associated with PTSD-like symptoms (Sojka et al. 2006). The above results indicate together that BBB impairment might be the crucial point of the generation of PTSD-related behaviors. However, a recent study presented results inconsistent with ours that the CSF/serum albumin ratio of SPS treated rats showed no distinct differences with control group (Dib et al. 2021). This may be attributed to the size of tracer molecules that albumin has a larger molecular weight than NaFlu (66458 Da vs. $376 \mathrm{Da}$ ).

Minocycline, a specific inhibitor of microglial activation, has been shown to present neuroprotective effects in various models of neurodegenerative diseases. Our previous study revealed that minocycline suppressed SPS-induced microglia activation in the hippocampus and attenuated both SPS-induced pain and anxiety-like behaviors (Sun et al. 2016). In this experiment, intraperitoneal injection of minocycline not only mitigated working memory impairment and improved the hippocampal BBB damage in SPS condition. Consistent with our studies, minocycline also attenuated hippocampal microglia activation and PTSD related behavioral changes including cognition, anxiety, and pain related parameters in other studies (Sun et al. 2016; Wang et al. 2018; Rooney et al. 2020). Several researches have documented that microglia-endothelial crosstalk is necessary for the regulation of BBB function. Wang et al. reported minocycline might preserve BBB via decreasing DKK1 expression in microglia involving Wnt1- $\beta$-catenin signaling (Wang et al. 2019). Wnt1- $\beta$-catenin pathway plays a crucial part in regulating the composition of endothelial tight junctions such as claudin 5, occludin, ZO-1 (Chang et al. 2017; Luo et al. 2020). Koichiro et al. reported that microglia enhanced BBB integrity via expression of claudin 5 in the early inflammatory response and impaired BBB function by phagocytosing astrocytic end-feet during the sustained inflammation stage (Haruwaka et al. 2019). The findings suggest microglia plays a dual role in the regulation of BBB integrity. These indicate that activated microglia, at least in part, involves in stress induced BBB remodeling.

Of course, there are some limitations to the study. Firstly, considering the potential hippocampal injury through local minocycline delivery, systemic administration was used in the study. Although this way is lack of tissue specificity, it avoids the impact on cognitive performance and BBB permeability detection. Secondly, though minocycline is a valid microglial inhibitor, some studies indicated minocycline can also act on astrocytes and possess systematic anti-inflammation effects (Keller et al. 2011; Lavin Plaza et al. 2020). Therefore, we will take more tissue and cell-specific strategies to further confirm the current results.

In summary $ه$ the present study indicates that BBB impairment may be the link between PTSD and cognitive dysfunction. Microglial activation may be responsible for BBB remodeling at the early stage of SPS. We hope our findings can bring a new perspective to the research field of PTSD-related cognitive dysfunction.

\section{Declarations}

\section{Acknowledgement}


All authors listed are grateful for the technical and instrument support provided by the Department of Scientific Research, Drum Tower Hospital. We also thank Dr. Liangyu Peng and Rui Dong for their kind suggestions about the analysis of immunofluorescence images.

\section{Author contribution}

Study concept and design: Zhengliang Ma, Wei Zhang and Juan Zhang. Analysis and interpretation of data and drafting of manuscript: Kun $\mathrm{Ni}$ and Jixiang Zhu. Experiment implementation: Kun Ni, Jixiang Zhu, Xuan Xu, Yue Liu, Shuai Yang, Yulin Huang, Rui Xu and Li Jiang.

\section{Funding}

This work was supported by the China National Natural Science Foundation $(81971044,82171225$, 81771142, 81870875).

\section{Availability of Data and Materials}

The data is provided upon request.

Ethics approval and consent to participate Animal experiments were performed according to protocols approved by the Ethics Committee of Drum Tower Hospital, and all efforts were made to minimize the number of animals used and their suffering.

Consent for publication All authors listed agree to publish this article.

Competing interests The authors declare that they have no conflict of interest.

\section{Data availablility statement}

The datasets generated and/or analyzed during the current study are available from the corresponding authors on reasonable request.

Availability of data and materials The data is provided upon request.

\section{References}

BISHT K, OKOJIE K A, SHARMA K, et al (2021) Capillary-associated microglia regulate vascular structure and function through PANX1-P2RY12 coupling in mice. Nat Commun 12:5289. doi:10.1038/s41467-02125590-8.

BOWMAN G L, DAYON L, KIRKLAND R, et al (2018) Blood-brain barrier breakdown, neuroinflammation, and cognitive decline in older adults. Alzheimers Dement 14:1640-1650. doi:10.1016/j.jalz.2018.06.2857.

BROWN L A, CHEN S, NARINE K, et al (2020) DSM-5 PTSD symptom clusters and suicidal ideation in veterans. Psychiatry Res 288:112942. doi:10.1016/j.psychres.2020.112942. 
CHANG J, MANCUSO M R, MAIER C, et al (2017) Gpr124 is essential for blood-brain barrier integrity in central nervous system disease. Nat Med 23:450-460. doi:10.1038/nm.4309.

COMPEAN E, HAMNER M (2019) Posttraumatic stress disorder with secondary psychotic features (PTSDSP): Diagnostic and treatment challenges. Prog Neuropsychopharmacol Biol Psychiatry 88:265-275. doi:10.1016/j.pnpbp.2018.08.001.

DELANEY C, FARRELL M, DOHERTY C P, et al (2021) Attenuated CSF-1R signalling drives cerebrovascular pathology. EMBO Mol Med 13:e12889. doi:10.15252/emmm.202012889.

DIB P, ZHANG Y, IHNAT M A, et al (2021) TNF-Alpha as an Initiator of Allodynia and Anxiety-Like Behaviors in a Preclinical Model of PTSD and Comorbid Pain. Front Psychiatry 12:721999. doi:10.3389/fpsyt.2021.721999.

DUCHON A, GRUART A, ALBAC C, et al (2020) Long-lasting correction of in vivo LTP and cognitive deficits of mice modelling Down syndrome with an a5-selective GABA(A) inverse agonist. British journal of pharmacology 177:1106-1118. doi:10.1111/bph.14903.

HAN F, XIAO B, WEN L (2015) Loss of Glial Cells of the Hippocampus in a Rat Model of Post-traumatic Stress Disorder. Neurochemical Research 40:942-951. doi:10.1007/s11064-015-1549-6.

HARUWAKA K, IKEGAMI A, TACHIBANA Y, et al (2019) Dual microglia effects on blood brain barrier permeability induced by systemic inflammation. Nat Commun 10:5816. doi:10.1038/s41467-019-13812$\mathrm{z}$.

IADECOLA C (2017) The Neurovascular Unit Coming of Age: A Journey through Neurovascular Coupling in Health and Disease. Neuron 96:17-42. doi:10.1016/j.neuron.2017.07.030.

KELLER A F, GRAVEL M, KRIZ J (2011) Treatment with minocycline after disease onset alters astrocyte reactivity and increases microgliosis in SOD1 mutant mice. Exp Neurol 228:69-79. doi:10.1016/j.expneurol.2010.12.010.

LAVIN PLAZA B, PHINIKARIDOU A, ANDIA M E, et al (2020) Sustained Focal Vascular Inflammation Accelerates Atherosclerosis in Remote Arteries. Arterioscler Thromb Vasc Biol 40:2159-2170. doi:10.1161/atvbaha.120.314387.

LI J, LIU Y, LIU B, et al (2019) Mechanisms of Aerobic Exercise Upregulating the Expression of Hippocampal Synaptic Plasticity-Associated Proteins in Diabetic Rats. Neural Plast 2019:7920540. doi:10.1155/2019/7920540.

LUO X, LI L, ZHENG W, et al (2020) HLY78 protects blood-brain barrier integrity through Wnt/ $\beta$-catenin signaling pathway following subarachnoid hemorrhage in rats. Brain Res Bull 162:107-114. doi:10.1016/j.brainresbull.2020.06.003. 
MONTAGNE A, NATION D A, SAGARE A P, et al (2020) APOE4 leads to blood-brain barrier dysfunction predicting cognitive decline. Nature 581:71-76. doi:10.1038/s41586-020-2247-3.

NATION D A, SWEENEY M D, MONTAGNE A, et al (2019) Blood-brain barrier breakdown is an early biomarker of human cognitive dysfunction. Nat Med 25:270-276. doi:10.1038/s41591-018-0297-y.

RAJAGOPAL N, IRUDAYANATHAN F J, NANGIA S (2019) Computational Nanoscopy of Tight Junctions at the Blood-Brain Barrier Interface. Int J Mol Sci 20. doi:10.3390/ijms20225583.

ROGERS J P, CHESNEY E, OLIVER D, et al (2020) Psychiatric and neuropsychiatric presentations associated with severe coronavirus infections: a systematic review and meta-analysis with comparison to the COVID-19 pandemic. Lancet Psychiatry 7:611-627. doi:10.1016/s2215-0366(20)30203-0.

ROONEY S, SAH A, UNGER M S, et al (2020) Neuroinflammatory alterations in trait anxiety: modulatory effects of minocycline. Translational Psychiatry 10:256. doi:10.1038/s41398-020-00942-y.

SáNTHA P, VESZELKA S, HOYK Z, et al (2015) Restraint Stress-Induced Morphological Changes at the Blood-Brain Barrier in Adult Rats. Front Mol Neurosci 8:88. doi:10.3389/fnmol.2015.00088.

SOJKA P, STåLNACKE B-M, BJöRNSTIG U, et al (2006) One-year follow-up of patients with mild traumatic brain injury: occurrence of post-traumatic stress-related symptoms at follow-up and serum levels of cortisol, S-100B and neuron-specific enolase in acute phase. Brain injury 20:613-620.

SUN R, ZHANG Z, LEI Y, et al (2016) Hippocampal activation of microglia may underlie the shared neurobiology of comorbid posttraumatic stress disorder and chronic pain. Mol Pain 12. doi:10.1177/1744806916679166.

VILLASEñOR R, LAMPE J, SCHWANINGER M, et al (2019) Intracellular transport and regulation of transcytosis across the blood-brain barrier. Cell Mol Life Sci 76:1081-1092. doi:10.1007/s00018-0182982-x.

WANG C, XU K, WANG Y, et al (2020) Spinal cannabinoid receptor 2 activation reduces hypersensitivity associated with bone cancer pain and improves the integrity of the blood-spinal cord barrier. Regional anesthesia and pain medicine 45:783-791. doi:10.1136/rapm-2019-101262.

WANG G, LI Z, LI S, et al (2019) Minocycline Preserves the Integrity and Permeability of BBB by Altering the Activity of DKK1-Wnt Signaling in ICH Model. Neuroscience 415:135-146.

doi:https://doi.org/10.1016/j.neuroscience.2019.06.038.

WANG W, WANG R, XU J, et al (2018) Minocycline Attenuates Stress-Induced Behavioral Changes via Its Anti-inflammatory Effects in an Animal Model of Post-traumatic Stress Disorder. Frontiers in Psychiatry 9. doi:10.3389/fpsyt.2018.00558. 
XU G, LI Y, MA C, et al (2019) Restraint Stress Induced Hyperpermeability and Damage of the Blood-Brain Barrier in the Amygdala of Adult Rats. Front Mol Neurosci 12:32. doi:10.3389/fnmol.2019.00032.

XUE R, WAN Y, SUN X, et al (2019) Nicotinic Mitigation of Neuroinflammation and Oxidative Stress After Chronic Sleep Deprivation. Front Immunol 10:2546. doi:10.3389/fimmu.2019.02546.

YANG M, RAINONE A, SHI X Q, et al (2014) A new animal model of spontaneous autoimmune peripheral polyneuropathy: implications for Guillain-Barré syndrome. Acta neuropathologica communications 2:5-5. doi:10.1186/2051-5960-2-5.

YANG R, DAIGLE B J, JR., MUHIE S Y, et al (2013) Core modular blood and brain biomarkers in social defeat mouse model for post traumatic stress disorder. BMC Syst Biol 7:80. doi:10.1186/1752-0509-7-80.

ZHOU Y-G, SHANG Z-L, ZHANG F, et al (2021) PTSD: Past, present and future implications for China. Chinese journal of traumatology = Zhonghua chuang shang za zhi 24:187-208. doi:10.1016/j.cjtee.2021.04.011.

\section{Figures}

a
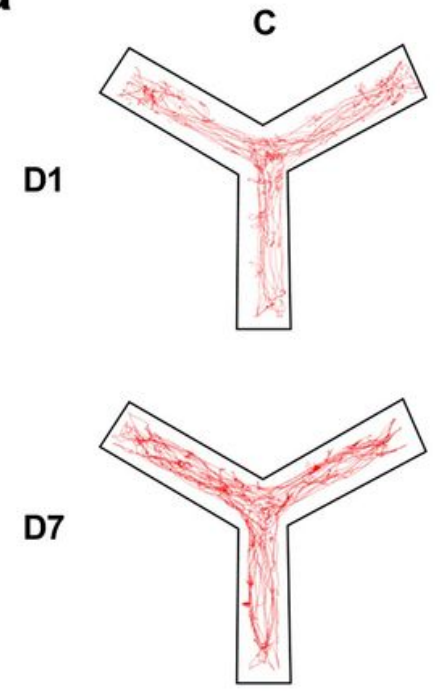
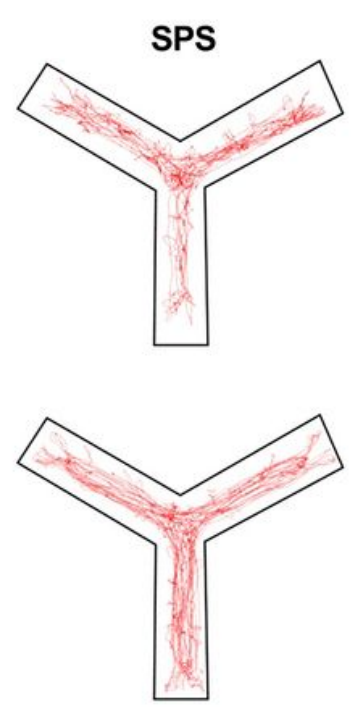

b

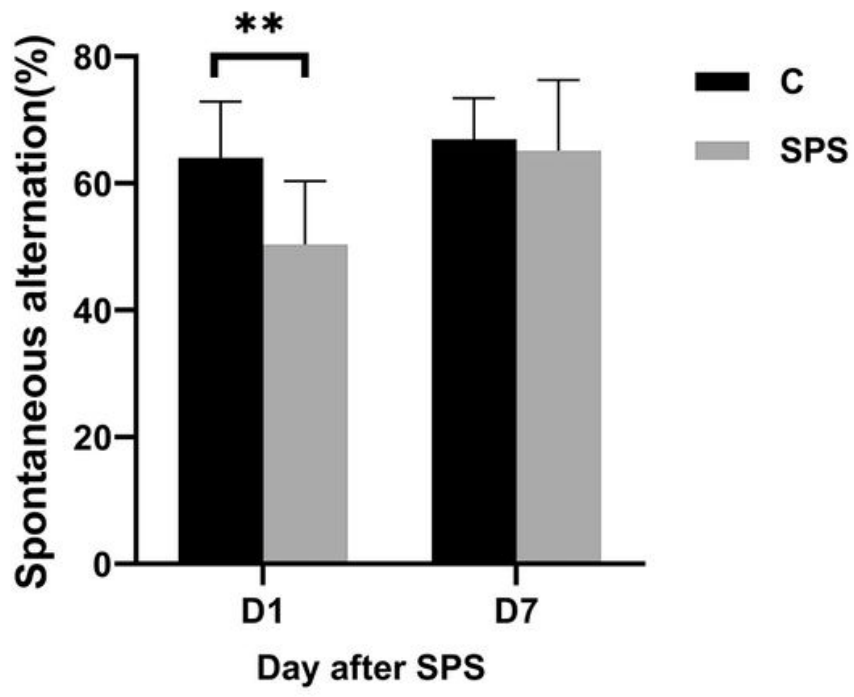

Figure 1

SPS induced cognitive dysfunction. a Representative rats' movement traces were presented on days 1 and 7 after SPS between group $C$ and group SPS. b Spontaneous alternation was tested on days 1 and 7 after SPS between group $C$ and group SPS. ${ }^{\star * P}<0.01$, compared to group $C$. 


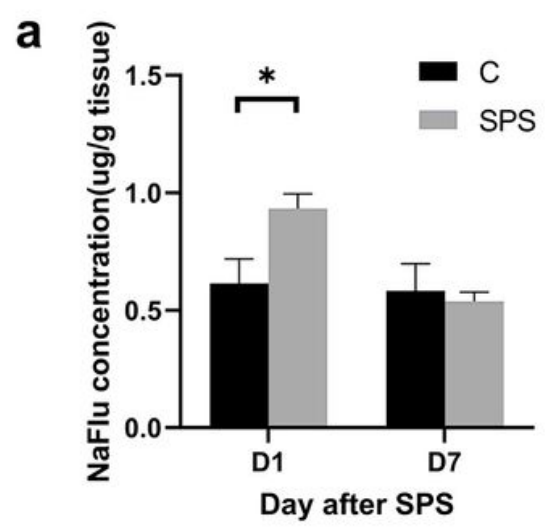

b
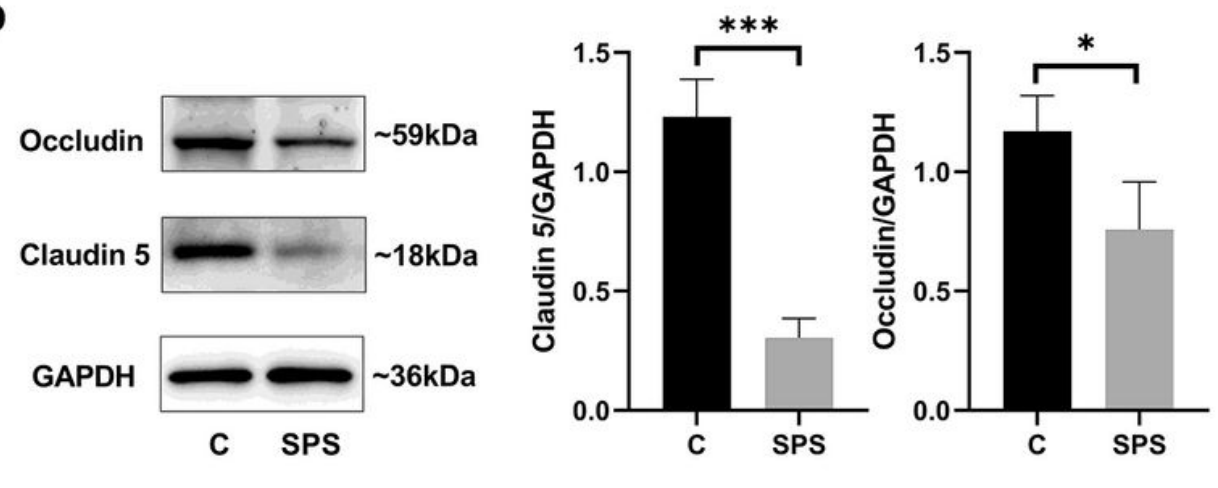

Figure 2

SPS induced hippocampal BBB impairment. a The NaFlu extravasation of hippocampus was tested on days 1 and 7 after SPS in each group ( $n=3$ /group). b Representative blots and quantification of tight junction proteins occludin and claudin 5 in the hippocampus on the first day after SPS ( $n=3 /$ group) *P< $0.05,{ }^{* *} \mathrm{P}<0.01,{ }^{\star * *} \mathrm{P}<0.001$. 


\section{a}

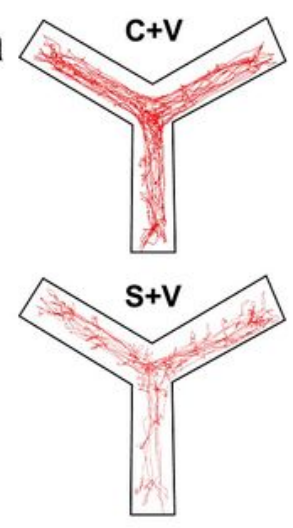

d
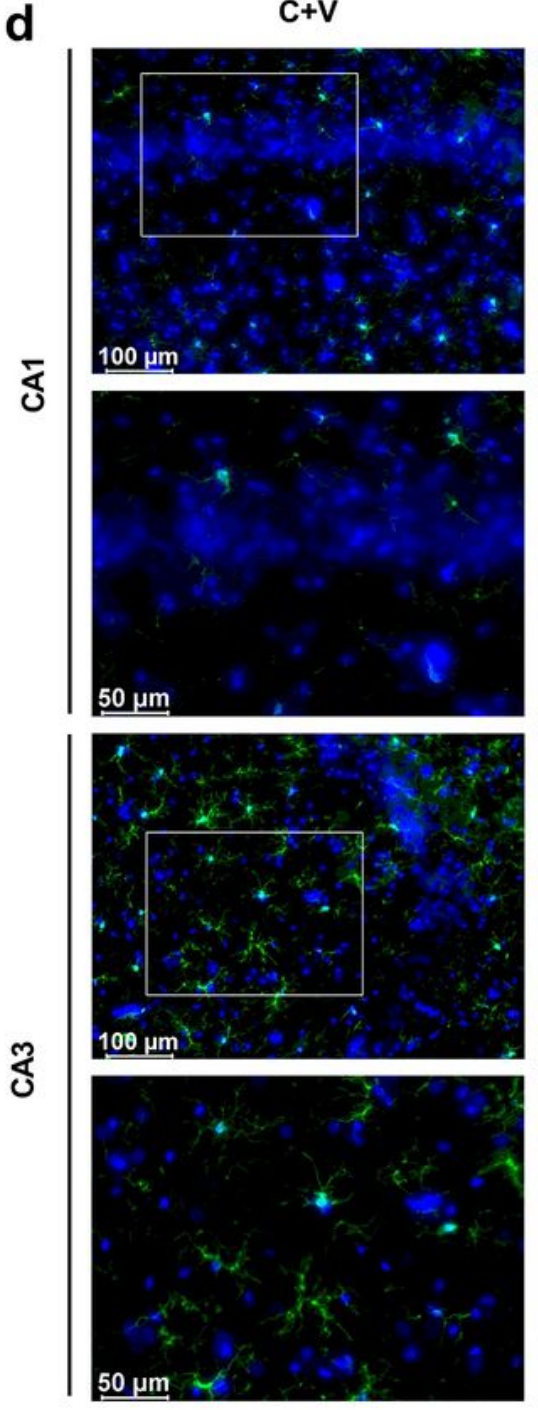

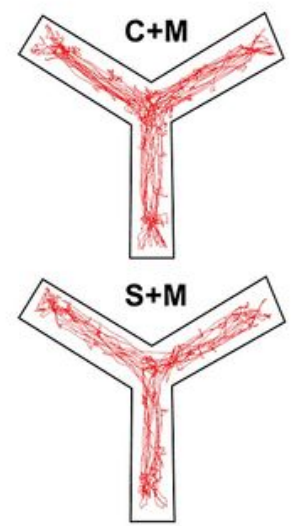

b

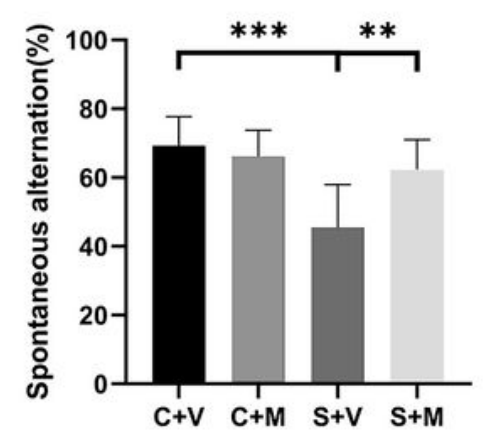

$\mathrm{C}+\mathrm{M}$
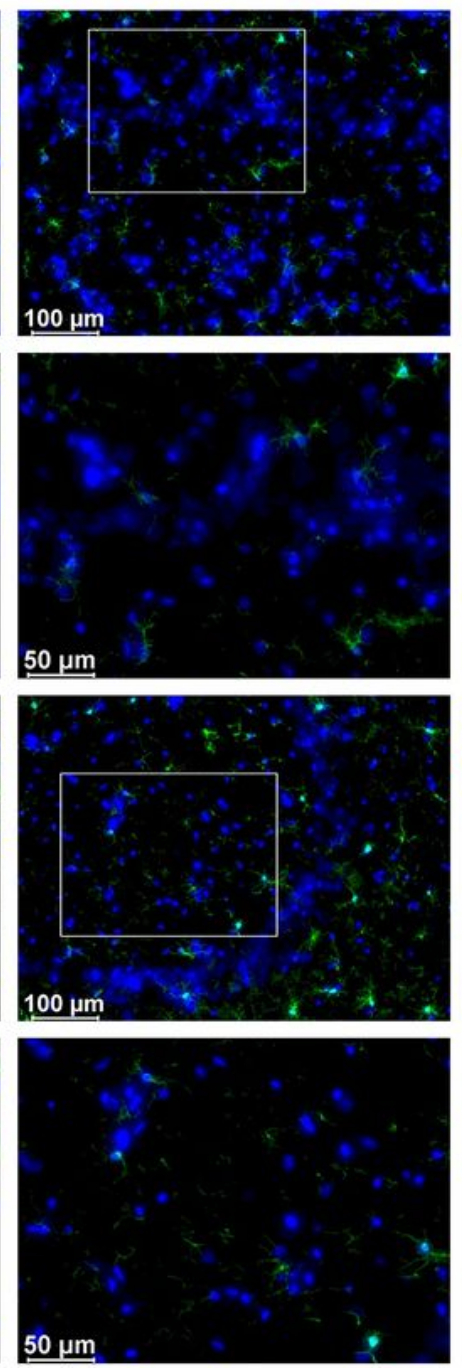

C

- $C+V \equiv C+M=S+V=S+M$

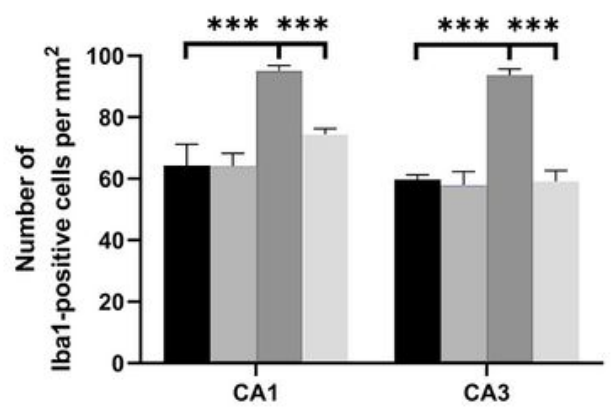

$S+V$

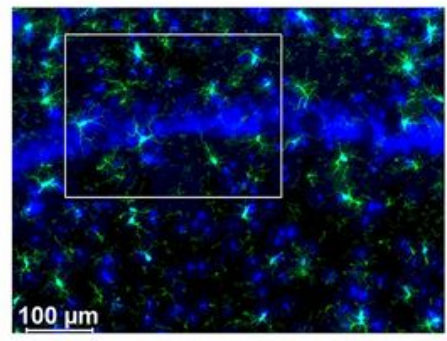

$100 \mu \mathrm{m}$
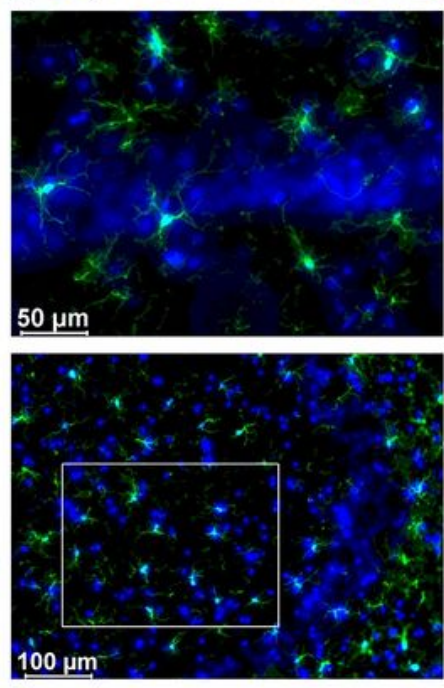

$50 \mu \mathrm{m}$

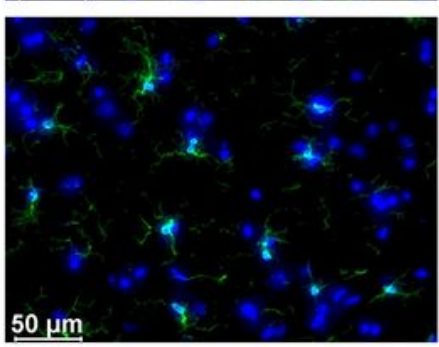

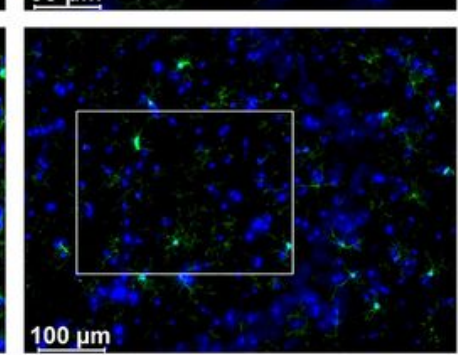

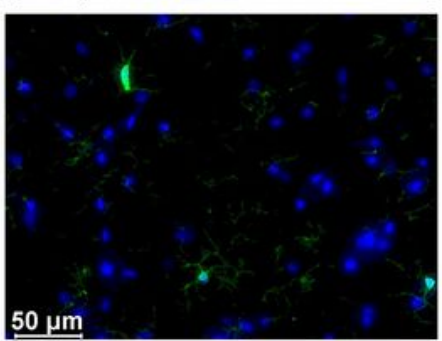

\section{Figure 3}

Inhibition of microglia attenuated cognitive impairment. a Typical trajectories were presented on days 1 after SPS in control and SPS exposed groups either treated with minocycline or vehicle intraperitoneally. $b$ Spontaneous alternation was tested on days 1 after SPS in control and SPS exposed groups either treated with minocycline or vehicle intraperitoneally ( $n=9 /$ group). c The number of lba1-positive cell bodies was summarized (6 fields of 3 rats/group). $d$ The representative Iba- 1 (green) and DAPI (blue) 


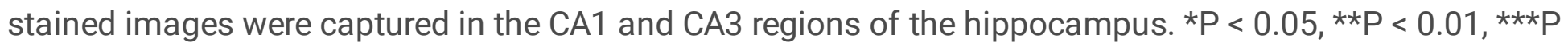
$<0.001$.

a

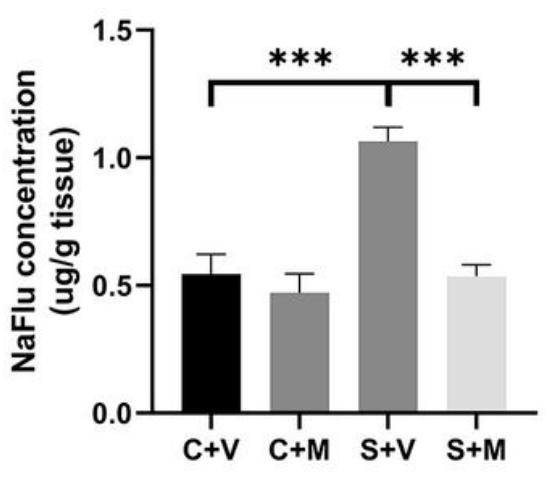

b

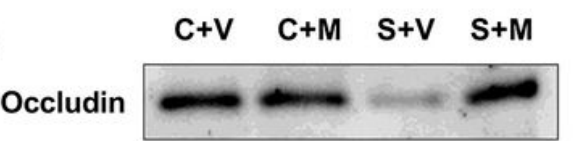

GAPDH

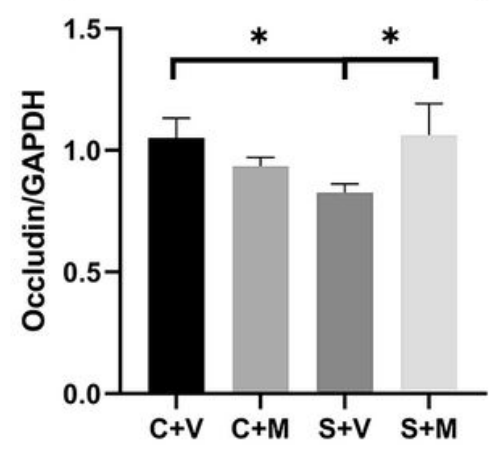

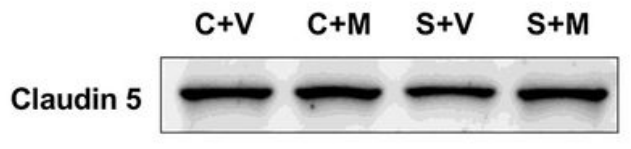

GAPDH
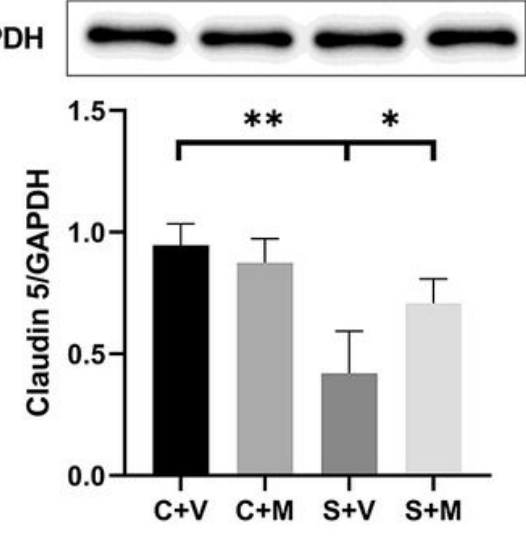

Figure 4

minocycline attenuated hippocampal BBB leakage. a The NaFlu extravasation in the hippocampus was tested on the day after SPS in in control and SPS exposed groups either treated with minocycline or vehicle intraperitoneally ( $n=3$ /group). b Representative blots and quantification of tight junction proteins levels of occludin and claudin 5 in the hippocampus on the first day after SPS were presented $(n=$ 3/group). ${ }^{*} P<0.05, * * P<0.01, * * * P<0.001$. 